\title{
Producción de artículos en la base de datos Web of Science y Scopus sobre educación física: estudio comparativo entre España y Brasil
}

\author{
Papers production in the database Web of Science and \\ Scopus about physical education: Comparative \\ study between Spain and Brazil
}

\author{
Joaquín REVERTER-MASÍA' \\ Vicenç HERNÁNDEZ-GONZÁLEZ' \\ Carme JOVE-DELTELL' \\ Teresa FONSECA² \\ Alejandro LEGAZ-ARRESE ${ }^{3}$
}

\section{Resumen}

Existe un creciente interés y necesidad en la evaluación de la producción científica. Entre las mediciones más importantes, está el número de artículos, el factor de impacto de la revista en que se publica y el índice de h. El objetivo analizar la producción de los profesores de las áreas de Educación Física de España y Brasil por medio de artículos en revistas en la base de datos Web of Science y Scopus. El método es un estudio descriptivo, comparativo y correlacional de análisis de documentos. La muestra la forman 373 profesores, de los cuales 108 corresponden a Brasil y 265 a España. Los resultados indican que los profesores en España tienen en Web of Science un Índice h 2.6 y 7.4 artículos por investigador y en Scopus una h 3.1 y 9.5 artículos. Los profesores brasileños en Web of Science tienen un Índice h de 6.05 y 30.91 artículos por autor y en Scopus una h 7.5 y 37.7 artículos. Las correlaciones entre número de artículos por profesor e Índice h son muy elevadas tanto en Web of Science como en Scopus. Las revistas en que más publican los profesores brasileños y españoles son Medicine and Science in Sports and Exercise y Journal of Strength and Conditioning Research. A modo de conclusión, el trabajo ha mostrado que existen notables diferencias de producción entre los profesores de los diferentes países. Los resultados muestran una mayor visibilidad de Scopus en relación a Web of Science.

Palabras Ilave: Deporte. Indicadores bibliométricos. Producción científica. Boletines. Scopus. Web of Science.

\begin{abstract}
There has been a growing interest and need in the evaluation of the quality and quantity of papers published by researchers. Therefore, the most important measurements are the number of items, the journal impact factor and the h-index. The aim of this paper is to analyze the publications of the professors in the field of Physical Education in Spain and Brazil published in journals in the Web of Science and Scopus databases. The method used is a descriptive, comparative and correlational analysis of documents. The sample consisted of 373 publications of professors of which 108 are in the field of Physical Education in Brazil and 265 in the field of Physical Education in

\footnotetext{
1 Universidad de Lleida, Faculdad de Educación, Departamento de Didácticas Específicas. Av. de l'Estudi General, 4E-25001, Lleida, España. Correspondencia a nombre de/Correspondence to:V.HERNÁNDEZ-GONZÁLEZ.E-mail:<vicens_h_g@didesp.udl.cat>.

2 Escola Superior de Educação, Comunicação e Desporto, Instituto Politécnico da Guarda, Departamento de Educação Física. Guarda, Portugal.

3 Universidad de Zaragoza, Facultad de Educación Física, Departamento de Fisiatría y Enfermería. Zaragoza, España.

Recibido el día 7/1/2013, re-presentado el 12/12/2013 y aceptado para su publicación el 27/2/2014.
} 
Spain. The results indicate that the professors in the field of Physical Education in Spain produced an $h$-index of 2.6 and 7.4 papers per researcher in Web of Science and an $h$-index of 3.1 and 9.5 papers per professor in Scopus. Brazilian professors obtained an h-index of 6.05 in Web of Science with a mean of 30.91 papers per researcher and $h$-index of 7.5 and 37.7 papers per professor in Scopus. Correlations between the number of papers per professor and h-index are high both in the Web of Science and Scopus. The majority of Brazilian and Spanish professors publish in Medicine and Science in Sports and Exercise and the Journal of Strength and Conditioning Research. Significant differences among professors of different countries were found in the present research.

Keywords: Sport. Bibliometric indicators. Scientific production. Journals. Scopus. Web of Science.

\section{Introducción}

La investigación como simple proceso de comprobación o refutación de los supuestos, teorías, fenómenos, es importante, ya que permite situar a un área del conocimiento a la vanguardia demostrando lo que tiene utilidad y lo que no. Estos hallazgos por sí solos no tienen relevancia si no se comunican, por lo tanto, la publicación de artículos es una actividad fundamental principalmente en las universidades y centros de investigación (Gil \& Sardinha, 2011). El fenómeno de la globalización se ha visto beneficiado por los avances en la comunicación, ha permitido un desarrollo más acelerado en la ciencia. Las revistas de calidad, por sus procesos de evaluación, son las incluidas en Web of science (WoS), ya que por tradición se han considerado las revistas de mayor prestigio (Olivas-Ávila \& MusiLechuga, 2010) y más recientemente las incluidas en Scopus (López-Illescas et al., 2008).

La evaluación de la productividad científica es un ejercicio necesario, ya que de esta manera los investigadores, los programas educativos y de manera global las universidades reciben ciertos beneficios a través de las evaluaciones de los organismos institucionales (García-Pérez, 2000).

Es un hecho constatado en que no existen parámetros de producción para algunas áreas del conocimiento (Carbonell \& Calvó, 2009), es decir, mientras se ha demostrado que existe una diferencia bastante amplia en función de la media de publicaciones que producen los profesores de diferentes áreas de conocimiento, como el área de Psicología Evolutiva y de la Educación (Musi-Lechuga et al., 2005), nunca se ha estudiado, que se tenga conocimiento, la producción del área de Educación Física (EF) en España y si existen diferencias entre diferentes países. Este problema nos lleva a plantearnos la necesidad de analizar la producción científica con el fin tener una evaluación objetiva del profesorado universitario (López, 2010).

La evaluación de la actividad científica y de la productividad de los investigadores es una cuestión de interés desde muchas perspectivas distintas: la financiación de la investigación, la promoción de los investigadores, la recompensa de la actividad de investigación, la formulación de políticas de investigación y la toma de decisiones relacionadas con tales políticas, la planificación estratégica de la actividad universitaria, la negociación salarial en los casos en que ésta se hace directamente entre el investigador y la organización contratante, la dotación de plazas de profesorado universitario y la promoción del profesorado y la concesión de becas, entre otras (Garcia-Pérez, 2000; Salgado \& Páez, 2007).

En estudios previos anteriores (Musi-Lechuga et al., 2005; Buela-Casal \&Zych, 2010) uno de los indicadores más fiables relacionados con la calidad académica de los profesores universitarios, son los registros en la WoS. Por otra parte, de manera complementaria, a partir de 2004 Elsevier puso en marcha Scopus, una base de datos que hoy indexa más de 16 mil revistas peer-reviewed, frente a las 11.500 de WoS (Thomson Reuters, 2010). Scopus es actualmente, por tanto, la mayor base de datos multidisciplinar existente (López-Illescas et al., 2008).

Con la aparición de Scopus aumenta la posibilidad de comparación, una característica crucial en la evaluación de la ciencia, que permite además los estudios complementarios a los que hasta ahora se habían realizado sobre los datos de WoS. Varios autores han centrado sus trabajos al comparar las características de ambas bases de datos teniendo en cuenta cuestiones como el precio, funcionalidades e interfaces de consulta (Goodman \& Deis, 2005). También han sido comparadas esas bases desde la perspectiva de su cobertura: títulos de revistas, áreas temáticas, idiomas, editores y 
distribución geográfica (Gorraiz \& Schloegl, 2008; LópezIllescas et al., 2008).

De modo general, dos aspectos de la actividad investigadora son tenidos en cuenta por los agentes evaluadores: la cantidad y la calidad de lo producido. No siempre estos indicadores han confluido, dando lugar a numerosos debates al respecto y posiciones encontradas. En el año 2005, el reputado físico Jorge Hirsch presentó el llamado " $h$-index", con el que sugería que se podía evaluar la actividad científica de los investigadores y que superaba las limitaciones de otros indicadores ampliamente utilizados para la clasificación de la productividad científica (Hirsch, 2005).

De acuerdo a Hirsch (2005, p.1),"Un científico tiene un índice $h$ si el $h$ de sus Np trabajos tienen al menos $h$ citas cada uno y los otros Np-h trabajos no tienen más que h citas cada uno". De modo más preciso, Rousseau (2006, p.1) señala que "El Índice de Hirsch es h si h es el rango más alto (número natural más alto) tal que las primeras $\mathrm{h}$ publicaciones reciben cada una al menos $\mathrm{h}$ citas." Por ejemplo, una h=20 significa que un autor tiene 20 publicaciones que han sido citadas cada una de ellas al menos veinte veces, pero no tiene 21 publicaciones que hayan sido citadas 21 veces cada una de ellas.

En opinión de Braun et al. (2005) entre las ventajas del Índice h se encuentran: a) que es robusto, es decir, es insensible a un exceso accidental de trabajos no citados y también a uno o varios extraordinariamente citados; b) que combina el efecto de la "cantidad" (número de publicaciones) y la "calidad" (tasa de citas) de un modo equilibrado y específico; y c) se puede calcular para períodos específicos y no sólo para toda una vida.

El índice $h$ ha sido aplicado a la literatura de la ciencia de la información por Cronin y Meho (2006), quienes encontraron, como era de esperar, una elevada y positiva correlación entre $h$ y el número de citas. Por otro lado, Aluja et al. (2011) muestran una gran diferencia en los científicos españoles de Psicología con un rango de valores del Índice $h$ que oscila entre 5 y 18.

El presente estudio tiene como finalidad hacer un análisis de la producción de los profesores de las áreas de Educación Física (EF) de España y Brasil por medio de artículos en revistas en la base de datos WoS y Scopus. Por lo tanto, los objetivos del presente estudio serán: presentar el índice $h$ de los profesores españoles y brasileños del área de conocimiento de EF. En segundo lugar, examinar la distribución estadística de dicho índice en esta población. En tercer lugar, examinar la relación que hay entre el índice $h$ y otras conocidas medidas utilizadas para evaluar la productividad investigadora, como el número de artículos y revistas en las que se publica. Y por último comparar la base de datos WoS y Scopus en el área analizada.

\section{Métodos}

Para la realización del estudio se utilizaron como fuentes de información las dos bases de datos especializadas en Ciencia y Tecnología de mayor relevancia para la comunidad internacional de académicos e investigadores: el WoS y Scopus.

La principal característica de estas dos bases de datos, y la que las convierte en herramientas imprescindibles para un análisis profundo de cualquier disciplina científica, es la posibilidad que ofrecen ambas de procesar las referencias bibliográficas de los artículos y cuantificar las citas recibidas por cada uno de ellos, lo cual permite la construcción de indicadores cualitativos para evaluar la actividad científica de una revista, el rendimiento científico de una institución o la visibilidad internacional de un autor determinado (Dorta-Contreras et al., 2008).

Se trata de un estudio descriptivo, comparativo y correlacional de análisis de documentos. Se tomó en cuenta las consideraciones propuestas por Hirsch (2005), las propuestas por Ramos-Álvarez et al. (2008) y el estudio de Olivas-Ávila y Musi-Lechuga (2010).

La muestra estuvo formada por todos los profesores con publicaciones en base de datos WoS en el área EF en España y profesores de la Plataforma Lattes, del área EF en Brasil.

Los instrumentos utilizados en este estudio fueron los siguientes: Páginas Web de los departamentos delárea de EF Española; Plataforma Lattes <http://buscatextual. cnpq.br/buscatextual/busca.do>; Base de datos WoS. Ésta se encuentra disponible a través de la plataforma |S| Web of Knowledge y contiene información sobre investigación multidisciplinaria en revistas para las cuales 
se calcula su Factor de Impacto (IF) y Base de datos Scopus. Ésta se encuentra disponible a través de la plataforma SCImago Journal Rank (SJR) y contiene información sobre investigación multidisciplinaria en revistas para las cuales se calcula su factor de impacto.

En primer lugar, se seleccionaron los profesores a partir de la información que aparecía en las páginas Web de los departamentos de EF de toda España (1.057 profesores delárea de EF) posteriormente se seleccionaron solamente los que tenían registros en WoS (265 profesores). En cuanto a los profesores brasileños se seleccionaron mediante la Plataforma Lattes, en ella, se aplicó un primer filtro relativo al "Área de Atuação", seleccionando como "Grande área"las" Ciências da Saúde" y como "Área" la "Educação Física". El segundo filtro aplicado fue relativo al estatus"Bolsistas de Produtividade do Conselho Nacional de Desenvolvimento Científico e Tecnológico (CNPq)" seleccionando las "Categorias/Nível de Bolsa" y seguidamente "1A, 1B, 1C, 1D y 2".

En segundo lugar los datos recabados se realizaron en función de los registros obtenidos en la WoS, teniendo en cuenta que en muchas ocasiones hay errores en los nombres de los autores (Ejemplo: Author= (Gonzalez, Luis-Millan) AND Address=(Univ Valencia)/ Author $=($ Gonzalez, LM o Author=(Casajus, Jose A.) AND Author=(Casajus, JA)). Se encontró que un mismo autor y un mismo trabajo citado, había sido recogido con nombres diferentes. Para una revisión más detallada del proceso es importante referirse al estudio de Osca-Lluch et al. (2009), donde se mencionan las posibles variaciones en los nombres y apellidos de los profesores y sus instituciones de afiliación. Por otra parte, otros acostumbran a unir sus apellidos por medio de un guión, por lo que también se tomó esto en consideración para la búsqueda.

El proceso de recolección de la información se realizó en la base de datos mencionada, ingresando en el apartado de "General Search", en donde se utilizó el campo de "Author" y "Address", incluso en algunos casos se utilizó el campo "Topic". Siempre se ingresaron uno o los dos apellidos del autor, algunos casos con letra mayúscula, en otros en minúscula, a veces seguidos por un espacio y las iniciales del nombre, también se emplearon truncadores y operadores booleanos para garantizar la obtención del total de artículos por autor considerando todas las combinaciones posibles en las que se pueden ordenar los apellidos, iniciales de los nombres e inicial del primer apellido, siguiendo en todos los casos el nombre suministrado por las webs oficiales de la Universidad o la Plataforma Lattes.

Una vez se localizaba cada autor con sus respectivos trabajos, se seleccionaba "Create Citation Report", para obtener el Índice h del autor. Para el estudio únicamente se seleccionaron aquellos profesores que tenían Índice h.

El proceso de recolección en Scopus se realizó ingresando en el apartado de "Author Search", donde se utilizó el campo de "Author"y"Affilation". Se ingresaron el primer apellido siempre con la letra mayúscula seguidos del segundo y separado en algunos casos por espacio y sus iniciales, también se emplearon truncadores y operadores booleanos para garantizar la obtención del total de artículos por autor considerando todas las combinaciones posibles en las que se pueden ordenar los apellidos, iniciales de los nombres e inicial del primer apellido, siguiendo en todos los casos el nombre suministrado por las webs oficiales de la Universidad o por la Plataforma Lattes.

Los resultados se acotaron por tipo de documento en este caso solo tomando en cuenta los etiquetados como artículos, revisiones y reimpresiones (article, review, reprint).

Las búsquedas de los profesores españoles, se llevó a cabo durante los meses de noviembre de 2011 hasta diciembre de 2011 por dos investigadores de manera independiente con la finalidad de obtener mayor fiabilidad de los resultados, mientras que este mismo proceso también se utilizó para la búsqueda de los profesores brasileños pero durante el mes de enero de 2012. Finalmente, se debe considerar que puede existir una variación al momento de consultar los resultados en el presente estudio y esto es debido principalmente a que la base de datos WoS no presenta reportes mensuales con resultados estáticos, por el contrario, se caracteriza por ser dinámica ya que se agrega información de manera constante, el número de registros puede variar de un día a otro. 


\section{Método de cálculo del índice $h$}

El método de cálculo del índice $h$ sugerido por Hirsch (2005) y descrito en más detalle por Imperial y Rodríguez-Navarro (2005) consiste en los siguientes pasos: 1) entrar en ISI Web of Knowledge y elegir la opción "General Search"; 2) escribir el nombre del autor en la ventana correspondiente; 3) cuando el sistema responda la lista de publicaciones, utilizar la opción de clasificación por orden de citas (Sort by times cited); y 4) a continuación identificar el último trabajo cuyo número de orden sea superior al del número de citas que tiene asociado. Ese número de orden corresponde al índice $h$ del autor.

En Scopus el cálculo consiste en los siguientes pasos: 1) Entrar en Scopus-Elsevier y elegir la opción "Search Author"; 2) escribir el apellido del autor en la ventana correspondiente "Last Name"; 3) cuando el sistema responda la lista de publicaciones, seleccionar el autor correspondiente; y 4) a continuación identificar en la ventana el $\mathrm{h}$ index del autor.

\section{Resultados}

Se analizaron un total de 373 profesores, de los cuales 108 corresponden al área EF de Brasil y 265 corresponden al área EF Española. En la base de datos WoS de los 108 profesores de Brasil 93 tenían Índice $h$ (86,11\%). Del área de EF en España de los 265 profesores, un total de 224 tenían Índice h (84,52\%). En Scopus 96 (88,88\%) profesores Brasileños tenían índice $h$, de los profesores Españoles 241 tenían índice h (90,90\%).

\section{El índice $h$ y número de artículos}

En la Figura 1 se muestra el índice $h$ medio que tiene de cada país en WoS y Scopus. Resaltar que en WoS

Profesores de España (WoS)

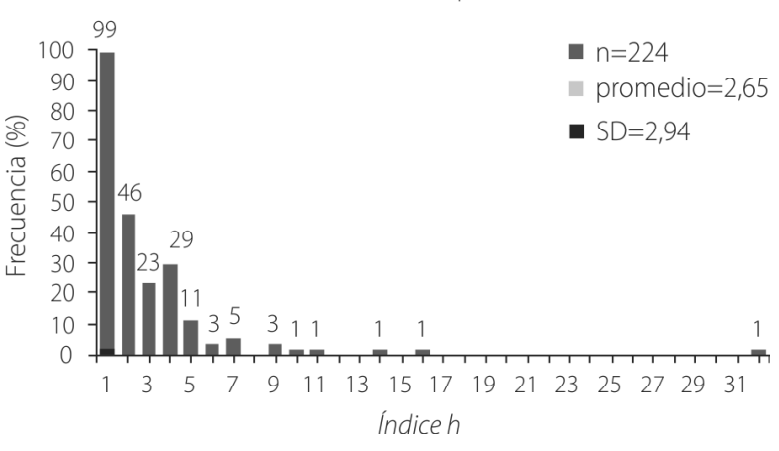

Profesores de Brasil (WoS)

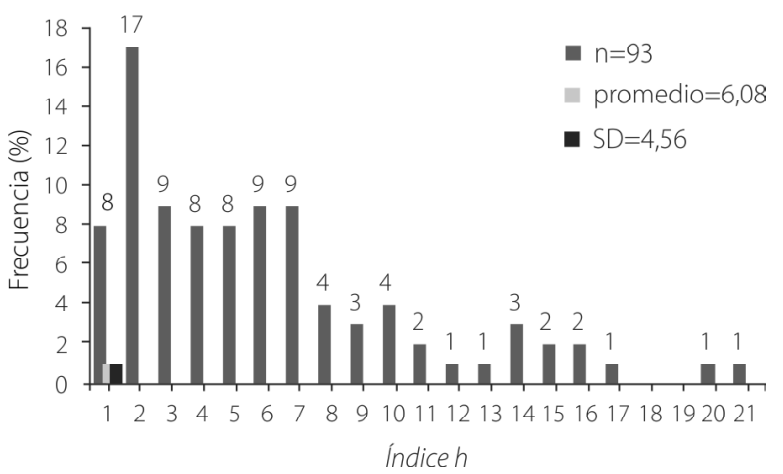

Profesores España (Scopus)

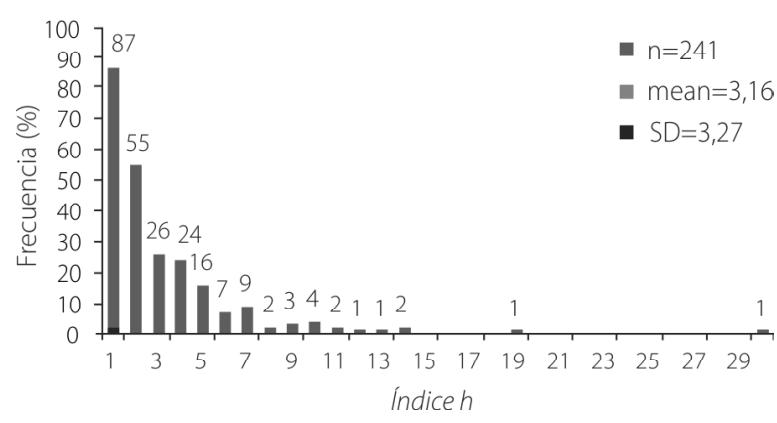

Profesores de España (Scopus)

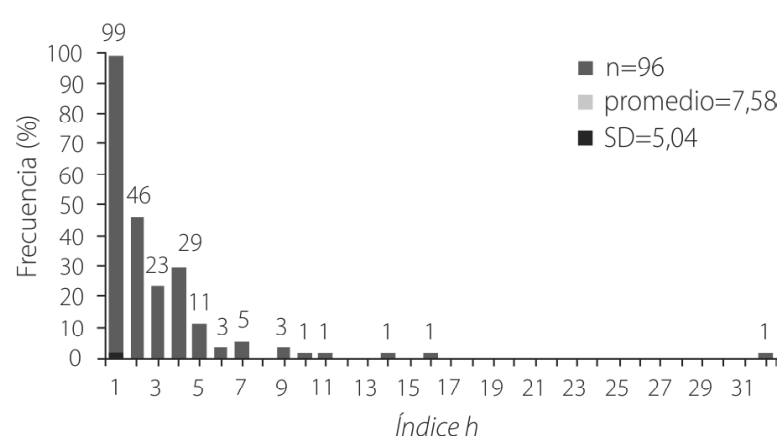

Figura 1. Indice $h$ de los profesores españoles y brasileños en Web of Science y Scopus. 
los profesores del área de EF en España tienen un índice h 2.65 (Figura 1) y una media de 7.49 artículos por profesores (Figura 2). En cuanto a los profesores de Brasil el rango de valores del índice hes 6.05 (Figura 1) y la media de artículos por profesor es de 30.91 (Figura 2).

En Scopus los profesores del área de EF en España tienen un índice h 3,16 (Figura 1) y una media de 9.5 artículos por profesor (Figura 2). En cuanto a los profesores de Brasil el rango de valores del índice hes 7,58 (Figura 1) y la media por profesor es de 37,72 (Figura 2).

\section{Convergencia entre los indicadores de la productividad y calidad científica}

En la Tabla 1 pueden verse las correlaciones entre los distintos indicadores utilizados en este estudio. De acuerdo con los objetivos, si atendemos a la relación del Índice $h$ con el total de de artículos por investigador. En ambos casos la magnitud de la correlación es muy elevada, tanto en WoS como en Scopus.

\section{Revistas con mayor número de registros de artículos por países}

Analizando la producción por países se encontró una gran variedad de revistas registradas en la base de datos WoS en las que publican los profesores. Observando la Tabla 2, se aprecia como las 3 revistas donde más comúnmente publican los profesores de EF de Brasil son en primer lugar la Medicine and Science in sports and Exercise con 171 artículos publicados, en segundo lugar la Journal of Strength and Conditioning Research con 159 entradas y en tercer lugar, la Revista
Profesores de España (WoS)

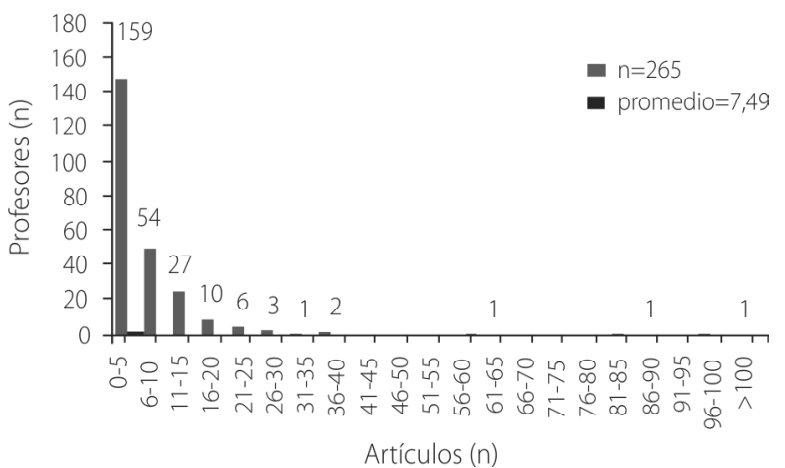

Artículos (n)

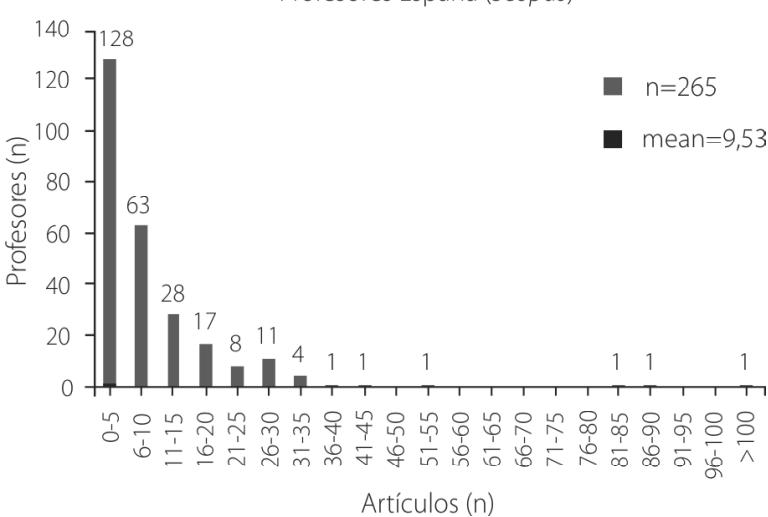

Profesores Brasil (WoS)
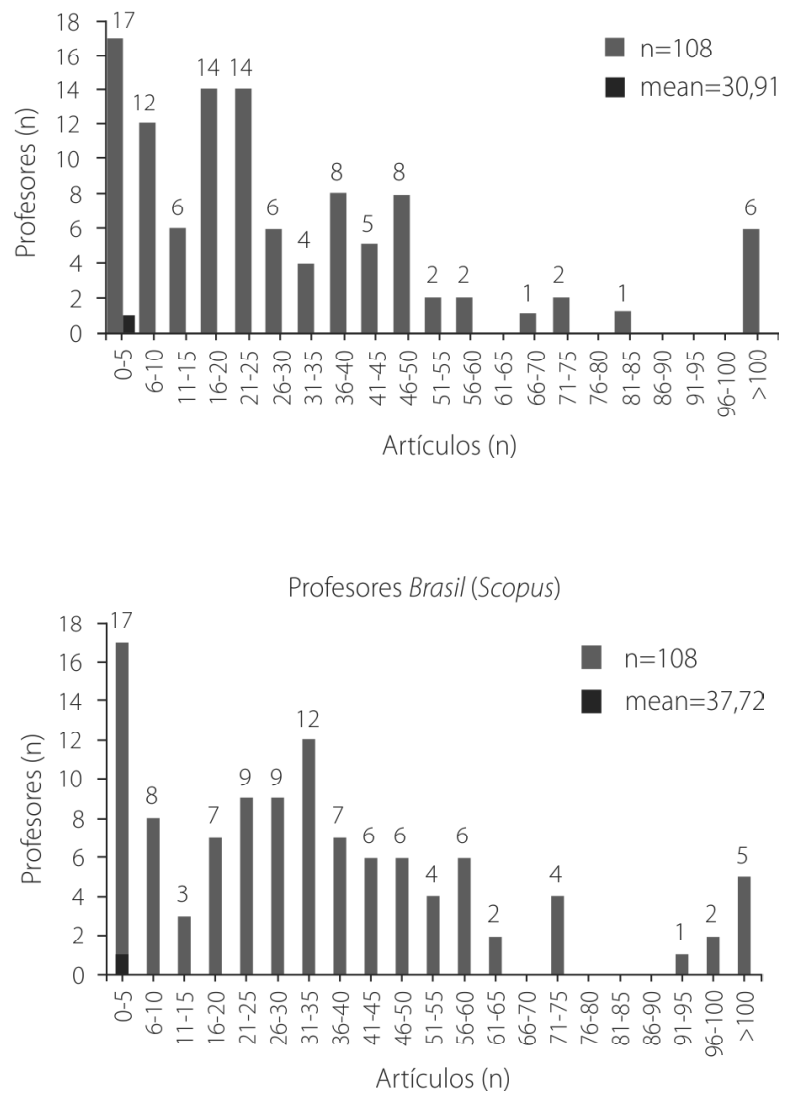

Figura 2. Número de artículos de los profesores españoles y brasileños en Web of Science y Scopus.

Fuente: De los autores (2013). 
Tabla 1. Correlaciones entre Indice $h$ y no de artículos en Web of Science y Scopus.

\begin{tabular}{|c|c|c|c|}
\hline \multicolumn{4}{|c|}{ Correlaciones } \\
\hline \multicolumn{2}{|c|}{ España } & \multicolumn{2}{|c|}{ Brasil } \\
\hline Índice h x NA (WoS) & Índice h x NA (Scopus) & Índice h x NA (WoS) & Índice h x NA (Scopus) \\
\hline $0,916667^{*}$ & $0,9066329^{*}$ & $0,867915^{*}$ & $0,8410211^{*}$ \\
\hline
\end{tabular}

Nota: ${ }^{*} p<.0001 ; h$ : Índice de Hirsch; NA=número total de artículos.

Fuente: De los autores (2013).

Tabla 2. Revistas registradas en la base de Web of Science con mayor número de registros en el área de Educación Física de España y Brasil.

\begin{tabular}{|c|c|c|c|c|c|c|c|}
\hline \multicolumn{4}{|l|}{ España } & \multicolumn{4}{|l|}{ Brasil } \\
\hline Revistas & Registros (n) & IF & Cuartil & Revistas & Registros (n) & IF & Cuartil \\
\hline $\begin{array}{l}\text { Journal of Strength and Conditioning } \\
\text { Research }\end{array}$ & 131 & 1.795 & Q2 & $\begin{array}{l}\text { Medicine and Science in Sports and } \\
\text { Exercise }\end{array}$ & 171 & 4.475 & Q1 \\
\hline Revista de Psicología del Deporte & 101 & 0.897 & Q3 & Journal of Strength and Conditioning & 159 & 1.795 & Q2 \\
\hline Medicine and Science in Sports and & 99 & 4.475 & Q1 & Research & & & \\
\hline Exercise & & & & Revista Brasileira de Medicina do & 156 & 0.268 & Q4 \\
\hline Perceptual and Motor Skills & 78 & 0.655 & Q4 & Esporte & & & \\
\hline $\begin{array}{l}\text { Journal of Sports Medicine and Physical } \\
\text { Fitness }\end{array}$ & 62 & 0.730 & Q3 & $\begin{array}{l}\text { Brazilian Journal of Medical and } \\
\text { Biological Research }\end{array}$ & 88 & 1.139 & Q3 \\
\hline European Journal of Applied Physiology & 56 & 2.660 & Q1 & Motriz Revista de Educação Física & 86 & 0.086 & Q4 \\
\hline Journal of Sports Science and Medicine & 55 & 0.885 & Q3 & Arquivos Brasileiros de Cardiologia & 80 & 1.130 & Q3 \\
\hline Internacional Journal of Sports Medicine & 51 & 2.268 & Q3 & Circulation & 80 & 15.202 & Q1 \\
\hline Journal of Sports Science & 42 & 2.082 & Q2 & European Journal of Applied & 67 & 2.660 & Q1 \\
\hline $\begin{array}{l}\text { Revista Internacional de Medicina y } \\
\text { Ciencias de la Actividad Física y del } \\
\text { Deporte }\end{array}$ & 37 & 0.205 & Q4 & $\begin{array}{l}\text { Journal of Sport Exercise } \\
\text { Psychology }\end{array}$ & 67 & 2.452 & Q1 \\
\hline Journal of Human Movement Studies & 37 & 0.056 & Q4 & Cadernos de Saúde Pública & 59 & 0.826 & Q4 \\
\hline European Journal of Sport Science & 29 & 1.146 & Q3 & $\begin{array}{l}\text { International Journal of Sports } \\
\text { Medicine }\end{array}$ & 57 & 2.268 & Q3 \\
\hline Internacional Journal of Obesity & 25 & 5.221 & Q1 & European Heart Journal & & 14097 & \\
\hline British Journal of Sports Medicine & 24 & 3.668 & Q1 & European Heart journal & 56 & $14.09 /$ & Q1 \\
\hline Haemophilia & 22 & 3.170 & Q2 & & 54 & 3.806 & Q1 \\
\hline International Journal of Sports & 23 & 0.867 & Q1 & Hypertension & 52 & 6.873 & Q1 \\
\hline Psychology & & & & Revista Brasileira de Fisioterapia & 50 & 1.000 & Q3 \\
\hline Nutrición Hospitalaria & 18 & 1.305 & Q4 & Revista de Saúde Pública & 46 & 1.066 & Q3 \\
\hline Journal of Applied Physiology & 17 & 3.484 & Q1 & Clinics & 43 & 2.058 & Q2 \\
\hline Archives of Physical Medicine and & 15 & 2.358 & Q1 & Faseb Journal & 38 & 5.704 & Q1 \\
\hline Rehabilitation & & & & Perceptual and Motor Skills & 37 & 0.655 & Q4 \\
\hline Psicothema & 13 & 0.961 & Q2 & Epilepsy Behavior & 36 & 1.844 & Q3 \\
\hline
\end{tabular}

Nota: IF: Factor de impacto.

Fuente: De los autores (2013).

Brasileira de Medicina do Esporte con 156 artículos. En cambio, los profesores de EF de España tienen en primer lugar la Journal of Strength and Conditioning Research con 131 artículos, en segundo lugar la Revista de Psicología del Deporte que tiene 101 entradas y en tercer lugar,
Medicine and Science in Sports and Exercise con más de 99 artículos.

En la Tabla 3 se aprecian las revistas donde más comúnmente publican segun la base de dstos Scopus. Los profesores de EF de Brasil publican en primer lugar 
Tabla 3. Revistas registradas en Scopus con mayor número de registros en el área de Educación Física de España y Brasil.

\begin{tabular}{|c|c|c|c|c|c|c|c|}
\hline \multicolumn{4}{|l|}{ España } & \multicolumn{4}{|l|}{ Brasil } \\
\hline Revistas & Registros (n) & $\mathrm{SJR}$ & Cuartil & Revistas & Registros (n) & SJR & Cuartil \\
\hline Archivos de Medicina del Deporte & 161 & 0.118 & Q4 & Revista Brasileira de Medicina do & 387 & 0.189 & Q4 \\
\hline Journal of Strength and Conditioning & 125 & 0.953 & Q1 & Esporte & & & \\
\hline Research & & & & Arquivos Brasileiros de Cardiologia & 168 & 0.267 & Q3 \\
\hline $\begin{array}{l}\text { Revista Internacional de Medicina y } \\
\text { Ciencias de la Actividad Física y del }\end{array}$ & 75 & 0.208 & Q2 & $\begin{array}{l}\text { Journal of Strength and Conditioning } \\
\text { Research }\end{array}$ & 147 & 0.953 & Q2 \\
\hline deporte & & & & Brazilian Journal of Medical and & 117 & 0.335 & Q2 \\
\hline Journal of Sports Medicine and Physical & 74 & 0.378 & Q3 & Biological Research & & & \\
\hline Fitness & & & & European Journal of Applied Physiology & 75 & 0.857 & Q2 \\
\hline Perceptual and Motor Skills & 78 & 0.257 & Q3 & Revista Brasileira De Cineantropometria & 73 & 0.000 & Q4 \\
\hline Revista de Psicologia del Deporte & 77 & 0.207 & Q3 & E Desempenho Humano & & & \\
\hline European Journal of Applied Physiology & 70 & 0.857 & Q2 & International Journal of Sports & 63 & 0.848 & Q2 \\
\hline Journal of Human Movement Studies & 71 & 0.558 & Q2 & Medicine & & & \\
\hline International Journal of Sport Medicine & 60 & 0.848 & Q2 & Cadernos de Saúde Pública & 56 & 0.880 & Q1 \\
\hline Journal of Sports Science and Medicine & 58 & 0.467 & Q1 & Revista de Saúde Pública & 54 & 1.070 & Q1 \\
\hline Journal of Sports Science & 55 & 0.909 & Q1 & Electromyography and Clinical & 49 & 0.167 & Q4 \\
\hline Medicine and Science in Sports and & 49 & 1.749 & Q1 & & & & \\
\hline Exercise & & & & $\begin{array}{l}\text { Medicine and Science in Sports and } \\
\text { Exercise }\end{array}$ & 48 & 1.749 & Q1 \\
\hline $\begin{array}{l}\text { Revista Andaluza de Medicina del } \\
\text { Deporte }\end{array}$ & 40 & 0.108 & Q4 & Clinics & 47 & 0.651 & Q1 \\
\hline Apunts Medicina de l'Esport & 39 & 0.146 & Q3 & Motriz Revista de Educação Física & 45 & 0.117 & Q4 \\
\hline European Journal of Sport Science & 40 & 0.444 & Q1 & Revista Brasileira de Fisioterapia & 42 & 0.279 & Q2 \\
\hline British Journal of Sports Medicine & 32 & 1.224 & Q1 & Epilepsy Behavior & 40 & 0.891 & Q1 \\
\hline International Journal of Obesity & 25 & 2.028 & Q1 & Perceptual and Motor Skills & 40 & 0.257 & Q3 \\
\hline Journal of Applied Psysiology & 24 & 1.359 & Q1 & Journal of Applied Physiology & 36 & 1.359 & Q1 \\
\hline Scandinavian Journal of Medicine and & 22 & 0.868 & Q2 & Arquivos de Neuro-psiquiatria & 33 & 0.276 & Q3 \\
\hline Science in Sports & & & & Cell Biochemistry and Function & 33 & 0.712 & Q2 \\
\hline Nutrición Hospitalaria & 22 & 0.263 & Q3 & International Journal of Cardiology & 28 & 0.712 & Q1 \\
\hline
\end{tabular}

Nota: SJR: SCImago Journal Country Rank. Fuente: De los autores (2013).

en la Revista Brasileira de Medicina do Esporte con 387 artículos publicados, en segundo lugar la Arquivos Brasileiros de Cardiologia con 168 entradas y en tercer lugar, la Journal of Strength and Conditioning Researchcon 147 artículos. En cambio, los profesores de EF de España tienen en primer lugar la Archivos de Medicina del Deporte con 161 artículos, en segundo lugar Journal of Strength and Conditioning Research que tiene 125 entradas y en tercer lugar, Revista Internacional de Medicina y Ciencias de la Actividad Física y del Deporte con más de 75 artículos.

\section{Discusión}

Este es el primer estudio, que se tenga conocimiento, que analiza el índice $h$ y las producciones científicas en las áreas de EF de diferentes países, WoS y Scopus. De la interpretación de los resultados que aquí se presentan se tiene que tener en cuenta el tipo de muestra utilizada, es decir, sus características y los criterios utilizados para realizar las clasificaciones. Siendo que se trata de la selección de todos los profesores elegidos de acuerdo a la información aparecida en una página Web resultaría difícil hacer una comparación con otros 
estudios, de otras áreas de conocimiento, en que las muestras son mucho menores.

Señala Ball (2005) que los procedimientos de elección de profesorado de las instituciones científicas son vistos a menudo como opacos, endogámicos y caprichosos, y que el método de Hirsch podría servir para informar la toma de decisiones de financiación o de promoción académica de un modo transparente, insesgado y difícil de falsear, porque descansa sobre el conjunto de trabajo realizado a lo largo de muchos años.

La presente investigación pone de manifiesto que existen importantes diferencias en la producción en artículos de revistas con factor de impacto en WoS y Scopus de los profesores de las áreas de EF de Brasil y España. Los profesores Brasileños producen muchos más artículos y tienen un Índice h mucho mayor que los Españoles. Según diferentes trabajos España y Brasil son los dos países con mayor producción científica en el Ranking Ibero-Americano (Marziale, 2011), sin embargo, en el área de EF la producción científica en España en mucho menor que en Brasil.

El presente estudio pone de relieve que la producción total en número de artículos es mucho mayor en Scopus que en WoS, posiblemente debido tal y como indica Santa y Herrero-Solana (2010) a que Scopus contiene una mayor cantidad de revistas fuente que permite identificar a priori un mayor número de citas, por lo tanto la visibilidad del trabajo es mucho mayor en Scopus que en WoS.

Cabe destacar que se ha encontrado una correlación muy positiva entre el Índice $h$ y la cantidad de artículos. Por lo tanto, en este caso la cantidad va relacionada con la calidad del investigador. Estos resultados se han producido por igual en las dos bases de datos analizadas.

Por otra parte, Hirsch propone un índice h de 20 después de veinte años de carrera como un indicador de buen rendimiento científico, en el caso de las Ciencias Físicas. Recordemos que la media de antigüedad de doctorado en ciencias del deporte en España es relativamente reciente. Por tanto, parece normal, que los españoles, tengan un índice inferior que los brasileños. Si observamos el Índice h, es mucho mayor en Scopus que en WoS. Teniendo presente que el Índice $h$ es una medición que se ha desarrollado para evaluar el impacto de un individuo en su campo de conocimiento. Los resultados encontrados corroboran los resultados obtenidos en otros estudios (Santa \& Herrero-Solana, 2010) sobre la inconveniencia de utilizar exclusivamente WoS como una fuente de datos bibliométricos, como se aprecia Scopus tiene una gran transcendencia y visibilidad en el ámbito científico analizado, por lo tanto la evaluación por parte de los organismos competentes (administraciones, agencias, gobiernos) no pueden limitarse exclusivamente a la WoS.

El índice h, cuya definición lleva implícito el reconocimiento de un grupo de artículos que determinan la efectividad de ese investigador, será mayor cuanto mayor sea la cantidad de artículos que formen parte de él (Roth, 2005). De esta forma, el orden de los autores más eficientes se transforma en el orden de los autores que mayor productividad y visibilidad han alcanzado de manera integral. Un autor muy productivo, pero con pocas citas recibidas, no es efectivo desde el punto de vista de Hirsch, así como tampoco lo será aquél que sólo produzca un artículo muy citado.

Si bien es cierto que existen algunos sesgos importantes en el cálculo del Índice h, como científicos que tienen una h muy elevada, el presente estudio nos ofrece una panorámica general de la situación de los dos países analizados.

Las revistas con factor de impacto en bases de datos reconocidas como criterio de evaluación de las instituciones universitarias es un hecho ampliamente estudiado, diferentes estudios establecen que la mayor parte de la producción científica se lleva a cabo por parte de un escaso número de profesores y investigadores (Sierra et al., 2008). Hay que tener presente que en España y Brasil existen muchos profesores universitarios que no tienen ninguna producción científica. Este hecho obliga a replantearse las funciones del profesor universitario en el siglo XXI y reflexionar si es necesario ser un buen investigador para ser un buen docente, a nuestro modo de entender estas dos cuestiones en el ámbito universitario son indivisibles.

Se ha observado una tendencia de los profesores universitarios a publicar en revistas de su propio país. En
121 
la base de datos WoS se registran cuatro revistas españolas en las que publican los profesores españoles y cinco en Scopus. En Brasil siete revistas brasileñas encontradas en WoS y nueve en Scopus. Por otra parte, se encontraron diferencias en cuanto al número de registros, siendo mucho mayor en Scopus. Esto reafirma las consideraciones de algunos informes internacionales acerca de las potencialidades de Scopus como futura herramienta de análisis y evaluación de la actividad científica, principalmente a partir de la aparición del portal SCImago Journal \& Country Rank, el cual utiliza los datos de Scopus para ofrecer un producto que emula con dos de los principales productos de Thomson Scientific: el Journal Citation Report y el Essential Science Indicators (Falagas et al., 2008).

El análisis comparativo entre los dos países pone de manifiesto que no hay costumbres de publicar muy diferentes. En este sentido cabe reseñar que las primeras revistas en los dos países, que tienen más concentración de artículos, son prácticamente las mismas el Journal of Strength and Conditioning Research y Medicine and Science in Sports and Exercise en WoS. Mientras que en Scopus de las tres primeras revistas dos son del propio país, lo cual reafirmaría la idea de la mejor cobertura regional de la base de datos Scopus.

Otro punto a tomar en consideración es la concentración de artículos en una misma revista, si analizamos las cuatro primeras revistas de España y Brasil, se observa que en la base de datos WoS estas cuatro revistas publican el 43,7\% de artículos realizados en España y el $38,7 \%$ de los realizados en Brasil. Si analizamos las cuatro primeras revistas de Scopus observamos que en España aglutinan el 36,8\% de los registros y en Brasil el 51,8\% de los artículos. Por lo tanto existe una concentración importante de artículos en revistas muy concretas en cada uno de los países.

Otro aspecto a tomar en cuenta de las revistas es el idioma de las mismas, esto puede llegar a condicionar la elección de las mismas a la hora de publicar, y en este sentido, en los últimos años han aparecido varias revistas de origen brasileño que aparecen en un lugar importante en la base de datos WoS como son la Revista Brasileira de Medicina do Esporte, Brazilian Journal of Medical and Biological Research o Arquivos Brasileiros de Cardiologia.
En el caso de España se encuentran la Revista de Psicología del Deporte y la Revista Internacional de Medicina y Ciencias de la Actividad Física y del Deporte. Posiblemente en el futuro ambos países tendrán más presencia de revistas en WoS y Scopus. Hay que tener en consideración que la mayoría de estas revistas busca una internacionalización y visibilidad de sus contenidos y actualmente publican sus trabajos en el idioma del país y en inglés.

En relación al idioma en que se publican los artículos, si bien es cierto que el inglés es la lengua vehicular en el ámbito científico, no se puede tolerar que esta lengua sirva de pretexto y permita que los países anglosajones hayan creado un monopolio de la comunicación científica (Quindos, 2009). Una parte importante de los mejores artículos de los grupos de investigación, se deberían publicar, bien en castellano o bien en inglés, en nuestras mejores revistas nacionales o supranacionales sin que ello conllevara una percibida menor calidad de la investigación. Al respecto, diferentes autores creen que deberían hacer una discriminación positiva de todos aquellos artículos escritos en lenguas científicas minoritarias que reciben un número no desdeñable de citaciones bibliográficas (Quindos, 2009).

Greenwood (2007) plantea que el factor de impacto puede ser análogo a un indicador del funcionamiento de una revista. Sin embargo, se debe ser muy cauteloso con la interpretación y el uso de las posiciones de las revistas en el ranking basado en el factor de impacto, puesto que las diferencias en la posición de una revista respecto a otra pueden deberse a diferencias mínimas en los factores de impacto respectivos. Matías-Gámez (2011) afirma que a la hora de evaluar los resultados de la actividad investigadora de grupos de investigación, instituciones o investigadores particulares de especialidades diferentes sería conveniente relativizar el IF de las revistas donde se publican tales resultados, utilizando para ello índices teniendo en cuenta las particularidades de la categoría o categorías donde se incluyen esas revistas. En nuestro estudio, se observa que el factor de impacto de las revistas españolas y brasileñas en WoS es relativamente bajo en su categoría. En Scopus las revistas, españolas y brasileñas, que tienen la mayor parte de los registros 
también tienen un IF bajo. Sin embargo, en el caso de Brasil hay tres revistas, con un número considerable de artículos, en el primer cuartil. Seria deseable que en el futuro las revistas más relevantes de cada país en el ámbito de la educación física se sometieran a las evaluaciones de las bases de datos analizadas con el fin de incrementar la presencia en el ámbito internacional y al mismo tiempo se incrementaría visibilidad de trabajos y las citaciones de las investigaciones realizadas en cada uno de los países analizados.

Con este trabajo hemos querido difundir entre la comunidad española y brasileña un instrumento útil para clasificar la productividad y la calidad de la investigación científica en la ciencia del deporte. Los resultados expuestos confirman la necesidad de investigaciones que profundicen aún más en las características de Scopus como herramienta de análisis de producción científica, y como alternativa firme a la base de datos WoS. Los resultados de esta investigación muestran una mayor visibilidad de Scopus en relación a WoS, por lo tanto ambas bases de datos deben ser complementarias, y no excluyentes, respecto a su posible utilización por los profesionales de la Educación Física.

\section{Conclusión}

En resumen, en este estudio se cumple con el objetivo de ofrecer una visión global de la producción científica de las ciencias del Deporte en WoS y Scopus en Brasil y España. De esta forma, los investigadores e instituciones conocen dentro del campo de la Educación Física la situación de algunos indicadores bibliométricos que influyen en el rendimiento científico. Estos trabajos deberían extrapolarse a las diferentes áreas, tanto en "Ciencias" como en "Ciencias Socia-les", ya que de esta forma los autores conocerían la situación de su campo de conocimiento correspon-diente, se aumentaría la visibilidad de las revistas y así se podría facilitar la mejor elección para publicar. Por otra parte, las instituciones pueden adoptar medidas que contribuyan a aumentar no solo la productividad, sino también la visibilidad.

\section{Referencias}

Aluja, A. et al. Indicadores de calidad de la producción en la Web of Science de diez profesores del área de Personalidad, evaluación y tratamiento psicológico: aportaciones adicionales al estudio de Olivas-Ávila y Musi-Lechuga. Psicothema, v.23, n.2, p.267-273, 2011. Disponible en: <http://www.redalyc.org/ articulo.oa?id=72717169016>. Acceso en: 6 feb. 2012.

Ball, P. An index for fair ranking of scientists. Nature, v.436, n.7053, p.900, 2005.

Braun, T.; Glänzel, W.; Schubert, A. A hirsch-type index fort journals. The Scientist, v.19, n.8, p.22, 2005. Available from: <http://sci2s.ugr.es/hindex/pdf/Braunetal2006.pdf>. Cited: Jun. 24,2008

Buela-Casal, G.; Zych, I. Analysis of the relationship between the number of citations and the quality evaluated by experts in Psychology journals. Psicothema, v.22, n.2, p.270-276, 2010.

Carbonell, X.; Calvó, N. Las revistas españolas de Psicología: cómo elegir la revista donde publicar. Anales de Psicología, v.25, n.2, p.209-216, 2009. Disponible en: <http://www.um.es/ analesps/v25/v25_2e.htm>. Acceso en: 15 abr. 2010.

Cronin, B.; Meho, L. Using the h-index to rank influential information scientists: Brief communication. Journal of the American Society for Information Science and Technology, v.57, n.9, p.1275-1278, 2006. Available from: <http://dl.acm.org/ citation.cfm?id=1144500.1144501 >. Cited: Oct. 11, 2011.
Dorta Contreras, A.J. et al. Productividad y visibilidad de los neurocientíficos cubanos: estudio bibliométrico del período 2001-2005. Revista de Neurología, v.47, n.7, p.355-360, 2008. Disponible en: <http://www.neurologia.com/pdf/Web/4707/ ba070355.pdf>. Acceso en: 12 abr. 2012.

Falagas, M. et al. Comparison of SCImago Journal Rank indicator with journal impact factor. Faseb Journal, v.22, n.8, p.2626-2628, 2008.

García-Pérez, M. Assessors' odd listings don't inspire confidence. Nature, v.406, n.6794, p.343, 2000.

Gil, C.; Sardinha, A. H-Index of the citing articles: A contribution to the evaluation of scientific production of experienced researchers. Revista Brasileira de Medicina do Esporte, v.17, n.5, p.358-362, 2011. Available from: <http://www.scielo.br>. Cited: Feb. 19, 2012

Greenwood, D. Reliability of journal impact factor rankings. BMC. Medical Research Methodology, v.48 n.7, p.48, 2007. Available from: <http://link.springer.com/article/10.1186\%2 F1471-2288-7-48\#page-1>. Cited: Nov. 15, 2009.

Goodman, D.; Deis, L. Web of Science (2004 version) and Scopus. Charleston Advisor, v.6, n.3, p.5-21, 2005.

Gorraiz, J.; Schloegl, C. A bibliometric analysis of pharmacology and pharmacy journals: Scopus versus Web of Science. Journal of Information Science, v.34, n.5, p.715-725, 2008. 
Hirsch, J.E. An index to quantify an individual's scientific research output. Proceedings of the National Academy of Sciences of the U.S.A, v.102, p.16569-16572, 2005. Available from: <http://www.pnas.org/content/102/46/16569.full.pdf+ html>. Cited: Aug. 25, 2009

Imperial, J.; Rodríguez-Navarro, A. La utilidad del índice $h$ de Hirsch para evaluar la investigación en España. 2005. Disponible en: <http://www.bit.etsia.upm.es/Imperial_ Rodriguez_Navarro.pdf>. Acceso en: 18 Mayo 2012.

López, L. Citación y dinámicas de la comunicación científica. Universitas Psychologica, v.9, n.1, p.9-12, 2010

López-Illescas, C.; Moya-Anegón, F.; Moed, H.F. Coverage and citation impact of oncological journals in the Web of Science and Scopus. Journal of Informetrics, v.2, n.4, p.304-316, 2008.

Marziale, M.H.P. Indicadores de la producción científica Iberoamericana. Revista Latino-Americana de Enfermagem, v.19, n.4, 2011. Disponible en: <http://www.scielo.br/pdf/rlae/ v19n4/es_01.pdf>. Acceso en: 4 nov. 2012.

Matías-Gámez, A. Relevancia de las revistas españolas incluidas en las bases de datos del Institute for Scientific Information medida a través del factor de impacto renormalizad. Investigación Bibliotecnológica, v.25, n.55, p.67-79, 2011.

Musi-Lechuga, B. et al. Producción de los profesores funcionarios de psicología en España en artículos de revistas con factor de impacto de la Web of Science. Psicothema, v.15, n.4, p.539-548, 2005. Disponible en: <http://digibug.ugr.es/ bitstream/10481/19569/1/20130788.pdf>. Acceso en: 24 jul. 2011.

Olivas-Ávila, J.A.; Musi-Lechuga, B. Análisis de la producción de los profesores funcionarios de Psicología en España en artículos de revistas de la Web of Science. Psicothema, v.22, n.4, p.909-916, 2010.

Osca-Lluch, J. et al. Consecuencias de los errores en las referencias bibliográficas: el caso de la revista Psicothema. Psicothema, v.21, n.2, p.300-303, 2009. Disponible en: <http://
www.psicothema.com/tabla.asp?Make $=2009 \&$ Team $=1002>$. Acceso en: 10 abr. 2010.

Quindós, G. Confundiendo al confuso: reflexiones sobre el factor de impacto, el índice h(Hirsch), el valor Q y otros cofactores que influyen en la felicidad del investigador. Revista Iberoamericana de Micología, v.26, n.2, p.97-102, 2009.

Ramos-Álvarez, M. et al. Criteria of the peer review process for publication of experimental and quasi-experimental research in Psychology: A guide for creating research papers. International Journal of Clinical and Health Psychology, v.8, n.3, p.751-764, 2008. Available from: <http://www.redalyc.org/ articulo.oa?id=33712016009>. Cited: Oct. 4, 2010.

Roth, D. The emergence of competitors to the Science Citation Index and the Web of Science. Current Science, v.89, n. 9, p.1531-1536, 2005

Rousseau, R. New developments related to the Hirsh index. 2006. Available from: <http://eprints.rclis.org/bitstream/ 10760/7616/1/Hirsch_new_developments.pdf>. Cited: Oct. 4, 2011 .

Salgado, J.; Páez, D. La productividad científica y el índice h de Hirchs [sic] de la psicología social española: convergencia entre indicadores de productividad y comparación con otras áreas. Psicothema, v.19, n.2, p.179-189, 2007.

Santa, S.; Herrero-Solana, V. Cobertura de la ciencia de América Latina y el Caribe en Scopus vs Web of Science. Investigación Bibliotecnológica, v.24, n.52, p.13-27, 2010.

Sierra, J. et al. Análisis transnacional del sistema de evaluación y selección del profesorado universitario. Interciencia, v.33, n.4, p.251-257,2008. Disponible en: <http://www.redalyc.org/pdf/ 339/33933404.pdf>. Acceso en: 12 dic. 2011.

Thomson Reuters. Web of Science coverage expansion. 2010. Available from: <http://community.thomsonreuters.com/t5/ Citation-Impact-Center/Web-of-ScienceCoverage-Expan Expansion/ba-p/10663>. Cited: Feb. 18, 2011. 
